\title{
Added value of multiple versus single sessions of repetitive transcranial magnetic stimulation in predicting motor cortex stimulation efficacy for refractory neuropathic pain
}

\author{
Benjamin Pommier, MD, MSc, ${ }^{1,4}$ Charles Quesada, MSc, ${ }^{3,4}$ Camille Fauchon, PhD, ${ }^{4}$ \\ Christophe Nuti, MD, PhD, ${ }^{1,4}$ François Vassal, MD, PhD, ${ }^{1}$ and Roland Peyron, MD, PhD 2,4
}

'Service de Neurochirurgie, ${ }^{2}$ Service de Neurologie, ${ }^{3}$ Centre d'Evaluation et de Traitement de la Douleur, Centre Hospitalier Régional Universitaire, Saint-Etienne; and 4INSERM U1028, UMR5292 Intégration Centrale de la Douleur chez l'Homme Centre de Recherche en Neurosciences de Lyon, Université Claude Bernard Lyon 1 \& Université Jean Monnet, Saint-Etienne, France

OBJECTIVE Selection criteria for offering patients motor cortex stimulation (MCS) for refractory neuropathic pain are a critical topic of research. A single session of repetitive transcranial magnetic stimulation (rTMS) has been advocated for selecting MCS candidates, but it has a low negative predictive value. Here the authors investigated whether multiple rTMS sessions would more accurately predict MCS efficacy.

METHODS Patients included in this longitudinal study could access MCS after at least four rTMS sessions performed 3-4 weeks apart. The positive (PPV) and negative (NPV) predictive values of the four rTMS sessions and the correlation between the analgesic effects of the two treatments were assessed.

RESULTS Twelve MCS patients underwent an average of 15.9 rTMS sessions prior to surgery; nine of the patients were rTMS responders. Postoperative follow-up was $57.8 \pm 15.6$ months (mean \pm standard deviation). Mean percentage of pain relief (\%R) was $21 \%$ and $40 \%$ after the first and fourth rTMS sessions, respectively. The corresponding mean durations of pain relief were respectively 2.4 and 12.9 days. A cumulative effect of the rTMS sessions was observed on both $\% R$ and duration of pain relief ( $p<0.01)$. The $\% R$ value obtained with MCS was $35 \%$ after 6 months and $43 \%$ at the last follow-up. Both the PPV and NPV of rTMS were $100 \%$ after the fourth rTMS session $(p=0.0045)$. A significant correlation was found between $\% R$ or duration of pain relief after the fourth rTMS session and \%R at the last MCS follow-up ( $R^{2}$ $=0.83, p=0.0003$ ).

CONCLUSIONS Four rTMS sessions predicted MCS efficacy better than a single session in neuropathic pain patients. Taking into account the cumulative effects of rTMS, the authors found a high-level correlation between the analgesic effects of rTMS and MCS.

https://thejns.org/doi/abs/10.3171/2017.12.JNS171333

KEYWORDS rTMS; repetitive transcranial magnetic stimulation; MCS; motor cortex stimulation; neuropathic pain; central pain

$\mathrm{C}$ ENTRAL (neuropathic) pain is a disabling condition occurring after $1.5 \%-8 \%$ of strokes and $10 \%-30 \%$ of spinal cord injuries. ${ }^{6,10,11}$ Since the introduction of electrical epidural motor cortex stimulation (MCS) in the early $1990 \mathrm{~s},{ }^{35}$ many prospective and retrospective open case series ${ }^{13}$ have further promoted MCS for alleviation of neuropathic pain. However, controlled studies ${ }^{21,29,37}$ have failed to prove the analgesic efficacy of MCS for various reasons, including the basic aspects of indication and patient selection. One specific major limitation was the lack of predictive factors for efficacy to guide clinicians regarding patient eligibility for MCS. Various clinical features have been investigated, such as pain characteristics, etiology and anatomical location of lesions, preoperative motor status, and time interval to surgery, but none has offered a clear prediction of MCS efficacy. ${ }^{30}$ Also, pharmacological

ABBREVIATIONS LEP = laser evoked potential; MCS = motor cortex stimulation; MEP = motor evoked potential; NPV = negative predictive value; NRS = Numerical Rating Scale; PPV = positive predictive value; ROC = receiver operating characteristic; rTMS = repetitive transcranial magnetic stimulation; SSEP = somatosensory evoked potential; \%R MCS = percentage of pain relief following MCS; \%R rTMS = percentage of pain relief following rTMS.

SUBMITTED May 31, 2017. ACCEPTED December 5, 2017.

INCLUDE WHEN CITING Published online May 18, 2018; DOI: 10.3171/2017.12.JNS171333. 
tests have been left out of patient screening even though the analgesic response to morphine, ketamine, amytal, ${ }^{38}$ and propofol $^{8}$ has been proposed for this purpose. To date, the most satisfactory predictor of MCS efficacy has remained a single session of repetitive transcranial magnetic stimulation (rTMS), with a reported positive predictive value (PPV) of more than $90 \%$ but a negative predictive value (NPV) of less than $70 \% .^{3,23}$

Interestingly, it was recently demonstrated that multiple sessions of rTMS can enhance or even initiate pain relief in a delayed manner, through a cumulative effect during the first four sessions. ${ }^{17,33}$ Given these findings, we hypothesized that multiple sessions of rTMS might predict MCS efficacy better than a single session. To address this question, we reviewed prospectively collected data in a series of patients with refractory neuropathic pain first treated with rTMS, then with MCS.

\section{Methods Patients}

All patients included in this retrospective study had suffered from typical drug-resistant, unilateral central (i.e., originating from a lesion of the central nervous system) neuropathic pain with severe intensity (Numerical Rating Scale [NRS] > 6/10) for at least 1 year. In the period between January 2010 and November 2015, patients were first treated with at least four sessions of rTMS, after which they could opt for either additional rTMS sessions or MCS. After the rTMS sessions, MCS was considered an option regardless of the efficacy of rTMS on pain relief. In previous research, ${ }^{33}$ we demonstrated a cumulative analgesic effect across the first four rTMS sessions, but no further pain relief was observed thereafter. Thus, the fourth session was considered the best time point to assess rTMS efficacy.

For each patient, we systematically collected 1) demographic data (age, sex), 2) clinical determinants of pain (etiology, presence or absence of allodynia and hyperpathia, duration of symptoms), and 3) neurophysiological status (laser evoked potentials [LEPs] and somatosensory evoked potentials [SSEPs], classified as "normal," "impaired," or "abolished"). Patients with ongoing depression, mood disorders, opioid treatments, or personality disorders (as assessed in a multidisciplinary pain center including psychiatric examination) were not included in the study.

Data had been prospectively collected without randomization according to a pre-established open-label therapeutic protocol.

\section{rTMS Procedure}

A $1 \times 1 \times 1-\mathrm{mm}$ slice thickness 3D T1-weighted MRI sequence was used to define the target of rTMS. A trained operator first localized the somatotopic representation of the hand over the primary motor cortex contralateral to the pain. The operator identified the omega-shaped knob of the central sulcus on axial sections to use it as the target and produce a maximal motor evoked potential (MEP). The coil was then moved toward the somatotopic representation of the painful area according to the classic homunculus anatomy. ${ }^{32}$ This was done using the neuronavigation system, moving along the central sulcus and facing the superior frontal gyrus (F1) for lower limb pain, the middle frontal gyrus (F2) for upper limb pain and hemibody pain, and the lower frontal gyrus (F3) for facial pain. ${ }^{5}$ The target was confirmed by producing MEPs in the corresponding region of the body. Once the target was circumscribed, its stereotactic coordinates were fixed and saved for the present and next rTMS sessions.

Magnetic stimulation was delivered with a MagPro stimulator (Magventure Tonika Elektronic) through a figure-eight coil. To maintain the coil over the target throughout the rTMS session, its positioning was assisted by a robotic arm (Smartmove, ANT) coupled with a neuronavigation system (Visor2, ANT). This device allowed selection of the target and compensated (in real time) for head and body movements. Thus, once defined, the target was kept constant for the 26 minutes of the rTMS session, and as an advantage over classic rTMS procedures, almost $100 \%$ of the magnetic load was delivered over the target. Stimulation parameters were based on prior recommendations $^{19}$ and previous work. ${ }^{1,2,20,22,27,31}$ They consisted of 20 consecutive trains of 80 stimulations delivered at $20 \mathrm{~Hz}$, at $80 \%$ of the motor threshold, separated by intertrain intervals of 84 seconds (i.e., a total of 1600 stimulations in a 26-minute session).

We explained to patients that the effect of rTMS on their pain (responder or nonresponder status) was to be arbitrated after a test period made up of a series of four rTMS sessions separated by a 3- to 4-week interval. Thereafter, both nonresponders and responders could be engaged in an MCS program. While awaiting surgery, responders could continue noninvasive therapy based on maintenance rTMS sessions. The intervals between sessions were adapted to the patient's expectations and to the duration of the analgesic effect.

\section{MCS Procedure}

The surgical technique for MCS has been reported in detail elsewhere. ${ }^{30}$ In brief, two electrodes with four stimulating contacts (Resume, Medtronic) were sutured to the dura mater over the motor cortex via a frontoparietal craniotomy, with the patient under general anesthesia. The central sulcus was localized based on anatomical (3D MRI neuronavigation, Kolibri, BrainLab) and physiological (SSEP phase reversal technique) mapping. Electrode positioning was adapted according to the somatotopic representation of the painful area (Fig. 1), taking into account the previous optimal placement of the rTMS coil. Electrodes were connected to a dual-channel stimulator (PrimeAdvanced, Medtronic) subcutaneously implanted in the subclavicular region during the same operative stage.

Stimulation parameters were adapted during postoperative follow-up with the aim of optimizing the analgesic effect, based on our previous experience. ${ }^{30}$ The intensity of bipolar stimulation was gradually increased from 0.5 to $4.5 \mathrm{~mA}$ during the $1 \mathrm{st}$ month. The mean parameters of chronic stimulation were as follows: voltage $=2.1 \mathrm{~V}$ (range $0.5-4.5 \mathrm{~V})$; pulse duration $=186 \mu \mathrm{sec}($ range $70-300 \mu \mathrm{sec})$; frequency $=38 \mathrm{~Hz}($ range $30-60 \mathrm{~Hz})$; cyclic stimulation mode, 10 patients with 60 minutes on and 120 minutes off, and 2 patients with 30 minutes on and 30 minutes off. 


\section{Pain Assessment \\ Pain Assessment After rTMS}

At each rTMS session, a nurse (who was different from the operator delivering rTMS) evaluated the average pain relief declared by the patient, for the period of time from the previous rTMS session. The nurse was trained to do this using the criteria stated below, was blinded to the stimulation parameters, and collected the following variables as described by the patient. As previously proposed in chronic pain studies evaluating the efficacy of neuromodulation procedures, ${ }^{30,33}$ the percentage of pain relief following rTMS (\%R rTMS) as declared by the patients (pain relief scale ${ }^{18}$ ) was the primary outcome criterion. It is a subjective pain improvement score corresponding to a continuous $0 \%-100 \%$ scale based on the appraisal of pain intensity changes. Patients were asked to quantify the level of any pain relief between $0 \%$ (no pain relief) and $100 \%$ (complete pain relief) considering their basal pain level. Patients were also asked to quantify, as a secondary evaluation criterion, the duration of pain relief (i.e., the number of days during which they experienced pain relief). The other clinical variables collected were the pain intensity score, based on a 0-10 NRS at the beginning of each rTMS session, and changes in the level of analgesic drug intake (three conditions: unchanged, decreased, withdrawn).

Following the test period (first four rTMS sessions), patients were categorized according to $\% \mathrm{R}$ rTMS. Those who did not complete the four rTMS sessions or for whom $\% \mathrm{R}$ rTMS was $\leq 10 \%$ after the fourth rTMS session were characterized as nonresponders and advised to stop rTMS. Patients with \%R rTMS $>10 \%$ were categorized as responders and advised to continue rTMS therapy. As previously proposed in $\mathrm{rTMS}^{33}$ and $\mathrm{MCS}^{30}$ studies, responder patients were categorized as follows: low responders $(\% \mathrm{R}$ $=>10 \%$ to $39 \%)$, intermediate responders $(\% \mathrm{R}=40 \%-$ $69 \%)$, and high responders ( $\% \mathrm{R} \geq 70 \%)$. All patients could be referred to the neurosurgeon to undergo MCS.

\section{Pain Assessment After MCS}

Postoperative outcome was assessed with the same primary outcome criterion used for rTMS, that is, percentage of pain relief following MCS (\%R MCS). Assessment was systematically performed at 1 and 6 months after surgery, then every 6 months thereafter. Again, patients were categorized into low, intermediate, and high responders, according to \% RCS (same definition of thresholds as those for \%R rTMS). Stimulation parameters could be adapted in cases of insufficient relief or discomfort. The $\% \mathrm{R}$ MCS collected at the last follow-up was used as the main variable. The other clinical variables collected were pain score as assessed with the NRS; the computed percentage decrease in the postoperative NRS score; changes in the level of analgesic drug intake (three conditions: unchanged, decreased, withdrawn); the yes/no response to the question of whether the patient, assuming a similar level of MCS efficacy, would agree to undergo surgery again; and the treatment modality they preferred between multiple rTMS sessions and MCS (three conditions: equality, rTMS, MCS).
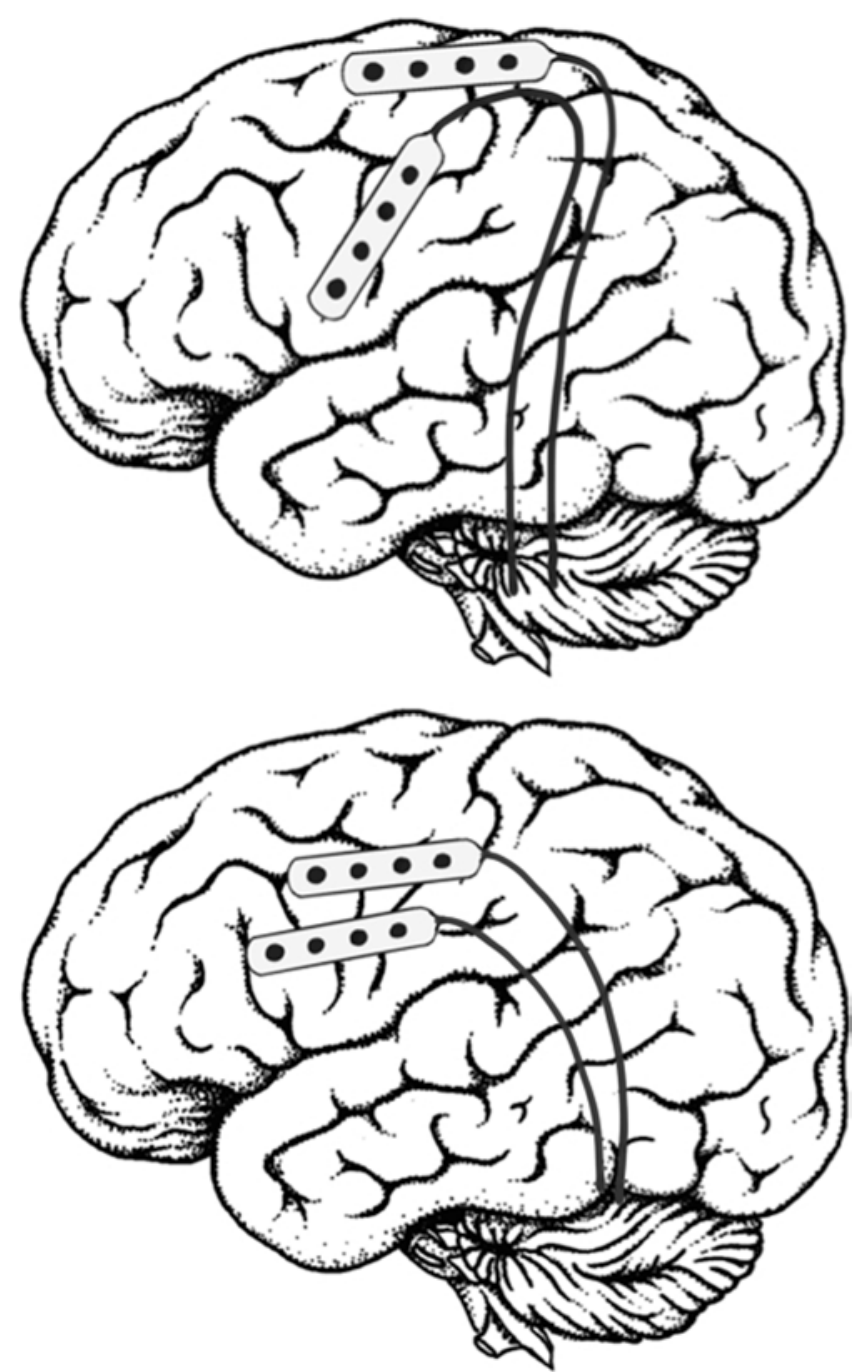

FIG. 1. Electrode positioning according to pain topography. A: Pain covering the whole hemibody: a first electrode was oriented anteroposteriorly, parallel to the parasagittal line, to treat pain in the lower limb. A second electrode was oriented superoinferiorly, along the main axis of the primary motor cortex, to treat pain in the face and/or upper limb. B: Pain covering the upper limb and face: the two electrodes were oriented anteroposteriorly, perpendicular to the primary motor cortex, in front of the corresponding somatotopic representations of the painful areas. Copyright Benjamin Pommier. Published with permission.

\section{Statistical Analysis}

Quantitative variables were expressed as the mean \pm standard deviation, and qualitative variables as the percentage of occurrence in the sample. Statistical significance was accepted at $\mathrm{p}<0.05$.

The effect of the number of rTMS sessions on $\% \mathrm{R}$ rTMS and rTMS duration of pain relief was assessed using nonparametric tests (Friedman test and post hoc Wilcoxon signed-rank test). The correlation between $\% \mathrm{R}$ rTMS and percentage decrease in postoperative NRS score was assessed using the Pearson correlation test.

After checking the normal distribution of the population (Shapiro-Wilk test), all the assumptions needed for the linear model were assessed: linearity and additivity of the relationship between dependent and independent 
TABLE 1. Clinical characteristics of 12 patients with unilateral central neuropathic pain

\begin{tabular}{|c|c|c|c|c|c|c|c|c|c|c|}
\hline \multirow{2}{*}{$\begin{array}{c}\text { Case } \\
\text { No. }\end{array}$} & \multirow[b]{2}{*}{ Sex } & \multirow{2}{*}{$\begin{array}{l}\text { Age } \\
\text { (yrs) }\end{array}$} & \multicolumn{4}{|c|}{ Pain Characteristics } & \multirow[b]{2}{*}{ Pain Topography } & \multirow[b]{2}{*}{ Lesion Type } & \multirow[b]{2}{*}{ SSEPS } & \multirow[b]{2}{*}{ LEPs } \\
\hline & & & Continuous & Paroxystic & Allodynia & Hyperpathia & & & & \\
\hline 1 & M & 59 & Yes & Yes & Yes & No & Lt face \& upper limb & Brainstem ischemic stroke & Normal & Abolished \\
\hline 2 & M & 66 & Yes & Yes & No & No & Lt thorax \& cervical regions & Spinal cord ischemia & Normal & Impaired \\
\hline 3 & $\mathrm{~F}$ & 44 & Yes & No & Yes & No & Lt hemibody & Perinatal lesion & Normal & NA \\
\hline 4 & $\mathrm{~F}$ & 38 & Yes & Yes & Yes & No & Amputation stump & Upper limb amputation & NA & NA \\
\hline 5 & M & 51 & Yes & Yes & Yes & Yes & Lt hemibody & Thalamic ischemic stroke & NA & Impaired \\
\hline 6 & M & 55 & Yes & No & Yes & No & Lt hemibody & Lobar ischemic stroke & Impaired & Abolished \\
\hline 7 & M & 78 & Yes & No & Yes & No & Lt hemibody & Lobar ischemic stroke & Impaired & NA \\
\hline 8 & M & 49 & Yes & No & Yes & No & Rt hemibody & Arnold-Chiari malformation & Impaired & Impaired \\
\hline 9 & M & 44 & Yes & No & Yes & No & Lt hemibody & Lacunar ischemic strokes & Impaired & Impaired \\
\hline 10 & M & 69 & Yes & Yes & Yes & No & Lt hemibody \& rt side of face & Brainstem ischemic stroke & Normal & Impaired \\
\hline 11 & $\mathrm{M}$ & 59 & Yes & Yes & Yes & No & Rt hemibody & Brainstem ischemic stroke & Impaired & Impaired \\
\hline 12 & M & 59 & Yes & Yes & Yes & Yes & Lt hemibody & Spinal cavernoma & Impaired & Abolished \\
\hline
\end{tabular}

$\mathrm{NA}=$ not available

variables, statistical independence of the error, homoscedasticity of the errors (Breusch-Pagan test), and normality of the error distribution (Shapiro-Wilk test).

The relationships between \%R rTMS or rTMS duration of pain relief (independent variables) and \% R MCS (dependent variable) were assessed using a linear regression model, first separately (simple linear regression model) and then together (multiple linear regression model).

A receiver operating characteristic (ROC) curve was plotted to assess the optimal \%R rTMS at the fourth session to predict MCS success. Its performance was assessed with a dichotomous predictive test (Mann-Whitney U-test and Youden's index). Once the optimal \%R rTMS threshold was established, contingency tables showing the relationship between rTMS and MCS results were drawn up to obtain an intermediate to good result with MCS. The significance of the contingency tables constructed to compare \%R rTMS and \% RCS was tested using Fisher's exact test.

The threshold for arbitrary definition of MCS success had previously been set to $30 \%$ of pain relief, ${ }^{3}$ but we considered a \%R MCS of $40 \%$ (i.e., intermediate responders) was the minimal goal to reach to justify invasive treatment. We, therefore, successively assessed the dichotomic predictive value of rTMS for both of these thresholds.

Finally, the level of analgesic drug intake was transformed into numeric values $(0=$ unchanged, $1=$ decreased, $2=$ withdrawn). Correlations between rTMS results and medication changes were assessed using Spearman's correlation test.

\section{Results}

\section{Population and Follow-Up}

The study included 12 patients ( 10 men, mean age 53.7 \pm 12.4 years, range $38-78$ years) who had suffered from unilateral central neuropathic pain with various etiologies. Clinical characteristics are summarized in Table 1. All the patients had been treated according to recommendations for chronic neuropathic pain (Table 2).

\section{rTMS Therapy}

Patients underwent a mean $15.9 \pm 12$ rTMS sessions $(\min =3, \max =33)$ between January 2010 and November 2015. From the beginning of rTMS until the last rTMS session, the mean follow-up was $57.8 \pm 15.6$ months (range 33-83 months). The interval between rTMS sessions was a mean $25 \pm 5.1$ days. Because of a lack of efficacy, one patient discontinued rTMS after three sessions and two patients after four sessions. At the end of the trial period, nine patients were categorized as rTMS responders and underwent an average of 20.5 additional rTMS sessions ( minimum $=3$, maximum $=33$ ).

\section{Intensity of Pain Relief}

The mean $\%$ R rTMS after the first and fourth rTMS sessions was $21 \% \pm 30 \%$ and $40 \% \pm 34 \%$, respectively. Three patients $(25 \%)$ were categorized as rTMS nonresponders (Table 3). The averaged percentage of pain relief (overall $\%$ R rTMS) among rTMS sessions was $30 \% \pm 23 \%$, range $0 \%-67 \%$ ) in the whole population of patients. Overall \%R rTMS in the responders group was $38 \% \pm 20 \%$ (range $10 \%-67 \%)$.

\section{Duration of Pain Relief}

The mean duration of pain relief after the first and fourth rTMS session was $2.4 \pm 4.6$ days and $12.9 \pm 9.5$ days, respectively. The averaged duration of pain relief was $11 \pm 7.7$ days (range $0-21.2$ days) in the whole population of patients. Duration of pain relief was $14 \pm 4.0$ days (range 9.8-21.2 days) in the responders group.

\section{Cumulative Effect of Multiple rTMS Sessions}

Among the nine rTMS responders, the Friedman test showed a cumulative effect of rTMS session repetition on both \%R rTMS $(\mathrm{p}=0.00048)$ and duration of pain relief $(p=0.0006)$. Post hoc tests showed that the first two rTMS sessions induced a significantly lower \%R rTMS and shorter pain relief duration than the ensuing rTMS sessions (Fig. 2). No such difference was observed during the subsequent rTMS sessions. 
TABLE 2. Medications used before treating patients with neuromodulation (rTMS and MCS)

\begin{tabular}{|c|c|c|c|c|c|c|}
\hline $\begin{array}{l}\text { Case } \\
\text { No. }\end{array}$ & $\begin{array}{c}\text { SSRI or SNRI } \\
\text { Antidepressants }\end{array}$ & Tricyclic Antidepressants & Antiepileptics & Benzodiazepines & Ketamine & $\begin{array}{l}\text { Level } 2 \& 3 \\
\text { Analgesics }\end{array}$ \\
\hline 1 & Escitalopram & Amitriptyline & Pregabalin, gabapentin & Clonazepam & No & No \\
\hline 2 & Duloxetine, citalopram & Amitriptyline & Pregabalin, lamotrigine & Clonazepam & No & No \\
\hline 3 & No & Amitriptyline & Gabapentin, lamotrigine & No & Yes & Yes \\
\hline 4 & No & Amitriptyline, clomipramine & Gabapentin, pregabalin & Clonazepam & Yes & Yes \\
\hline 5 & No & Amitriptyline & Pregabalin & Clonazepam & No & No \\
\hline 6 & Mirtazapine & No & Pregabalin, carbamazepine, levetiracetam & No & No & No \\
\hline 7 & No & Amitriptyline & Gabapentin, pregabalin & Clonazepam & Yes & No \\
\hline 8 & No & No & Pregabalin, carbamazepine & No & No & Yes \\
\hline 9 & No & Amitriptyline & Pregabalin & No & Yes & No \\
\hline 10 & No & Amitriptyline & Pregabalin, gabapentin, carbamazepine & Clonazepam & No & No \\
\hline 11 & Duloxetine & No & Pregabalin, gabapentin, topiramate & No & No & No \\
\hline 12 & Citalopram & No & Pregabalin, gabapentin & No & No & Yes \\
\hline
\end{tabular}

SSRI = selective serotonin reuptake inhibitor; SNRI = serotonin-norepinephrine reuptake inhibitor.

\section{Other Clinical Variables}

Mean NRS score was $7.29 \pm 0.95$ before rTMS and 6.46 \pm 2.27 after four rTMS sessions, but the difference between the two did not reach significance $(p=0.27)$. During the rTMS phase, two patients reduced and one patient (8\%) increased their drug intake, while medications remained unchanged in the other nine patients $(75 \%)$.

\section{rTMS Adverse Side Effects}

Except for nonspecific, slight, and short-term tensiontype headache, no adverse side effects were reported during the experiment.

\section{MCS Therapy}

Patients underwent surgery in the period from Feb- ruary 2012 to November 2015 . The mean postoperative follow-up was $31.4 \pm 14.4$ months (range 14-57 months). Outcomes are summarized in Table 3.

Intensity of Pain Relief

The detailed results of both \%R rTMS and \%R MCS for each patient are presented in Supplementary Fig. 1. At 6 months postsurgery, the mean \%R MCS was $35 \% \pm 25 \%$ (range 0\%-75\%).

At the last follow-up, three patients were MCS nonresponders (\%R MCS $3.3 \% \pm 5.8 \%$ ), seven were intermediate responders (\%R MCS $51.4 \% \pm 8.52 \%$ ), and two were high responders (\%R MCS $75 \% \pm 7.1 \%$; Fig. 3$)$. In the MCS intermediate and high responders group (\%R MCS $\geq$ $40 \%$ ), the mean percentage of pain relief was $46 \% \pm 19 \%$

TABLE 3. Clinical data after rTMS and MCS in 12 patients

\begin{tabular}{|c|c|c|c|c|c|c|c|c|c|}
\hline \multirow{2}{*}{$\begin{array}{l}\text { Case } \\
\text { No. }\end{array}$} & \multicolumn{2}{|c|}{$\%$ R rTMS } & \multirow{2}{*}{$\begin{array}{c}\% \text { R MCS } \\
6 \text { Mos }\end{array}$} & \multirow{2}{*}{$\begin{array}{l}\text { Last Postop } \\
\text { FU (mos) }\end{array}$} & \multirow{2}{*}{$\begin{array}{l}\text { \%R MCS } \\
\text { Last FU }\end{array}$} & \multirow{2}{*}{$\begin{array}{c}\% \text { Decrease in } \\
\text { Postop NRS Score }\end{array}$} & \multirow{2}{*}{$\begin{array}{l}\text { Preference: } \\
\text { rTMS vs MCS* }\end{array}$} & \multirow{2}{*}{$\begin{array}{c}\text { Yes/No } \\
\text { Response† }\end{array}$} & \multirow{2}{*}{$\begin{array}{l}\text { Change in Level of } \\
\text { Analgesic Intakef }\end{array}$} \\
\hline & 1st Session & 4th Session & & & & & & & \\
\hline 1 & 50 & 85 & 75 & 33 & 80 & 71 & MCS & Y & $\downarrow$ \\
\hline 2 & 0 & 20 & 50 & 41 & 50 & 38 & MCS & Y & $=$ \\
\hline 3 & 0 & 25 & 45 & 50 & 45 & 17 & $=$ & $Y$ & $=$ \\
\hline $4 \S$ & 0 & 0 & 0 & 57 & 0 & 0 & $=$ & $\mathrm{N}$ & $=$ \\
\hline $5 \S$ & 0 & 0 & 10 & 48 & 10 & -17 & MCS & Y & $=$ \\
\hline 6 & 0 & 50 & 60 & 25 & 70 & 57 & MCS & $Y$ & $\downarrow$ \\
\hline $7 \S$ & 80 & 0 & 0 & 24 & 0 & 0 & $=$ & $\mathrm{N}$ & $=$ \\
\hline 8 & 0 & 50 & 50 & 27 & 50 & 43 & MCS & Y & $\downarrow \downarrow$ \\
\hline 9 & 65 & 50 & 25 & 15 & 60 & 44 & MCS & $Y$ & $\downarrow$ \\
\hline 10 & 10 & 30 & 50 & 19 & 50 & 29 & MCS & Y & $\downarrow \downarrow$ \\
\hline 11 & 50 & 100 & 45 & 24 & 65 & 57 & MCS & Y & $\downarrow \downarrow$ \\
\hline 12 & 0 & 20 & 10 & 14 & 40 & 25 & MCS & $Y$ & $\downarrow$ \\
\hline
\end{tabular}

FU $=$ follow-up.

* Treatment modality preferred by patient (three conditions: equality, rTMS, MCS).

$\dagger$ Patient's answer to the question of whether they would agree, assuming a similar level of efficacy of MCS, to undergo surgery again.

$\ddagger$ Changes from the beginning of the treatment protocol to the last follow-up (three conditions): = unchanged, $\downarrow$ decreased, $\downarrow \downarrow$ withdrawn.

$\S$ Patients who did not benefit from either rTMS or MCS. 

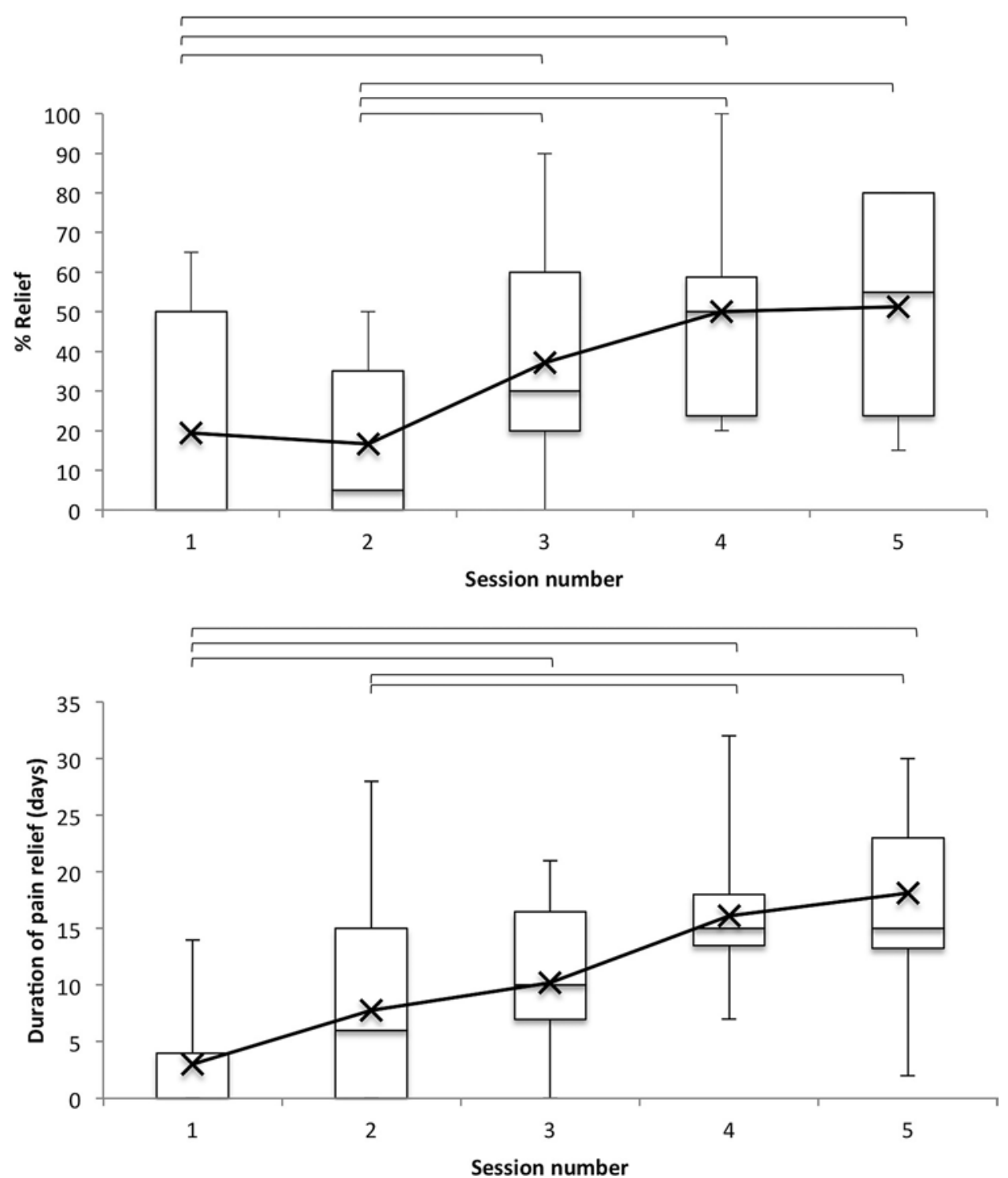

FIG. 2. Percentage (upper) and duration (lower) of pain relief according to rTMS session number in rTMS responders. Percentage of pain relief: Friedman test, $F=20.08, p=0.00048$. Duration of pain relief: Friedman test, $F=19.77, p=0.00055$. Square brackets indicate a significant Wilcoxon signed-rank post hoc test $(p<0.05)$.

(range 10\%-75\%). In the whole patient population, the mean $\%$ R MCS was $43 \% \pm 27 \%$ (range $0 \%-80 \%$ ), mean NRS score was $4.9 \pm 1.7$ (range 2-8), and mean percentage decrease in the postoperative NRS score was $30 \% \pm 27 \%$ (range 17\%-71\%). In the MCS responders group, mean $\%$ R MCS was $57 \% \pm 13 \%$ (range $=40-80 \%$ ), mean NRS score was $4.2 \pm 1.3$ (range 2-6), and mean percentage decrease in postoperative NRS score was $42 \% \pm 18 \%$ (range $17 \%-71 \%)$. A correlation was observed between \%R MCS and percentage decrease in postoperative NRS score $(\mathrm{R}=$ 0.94, $p=7.9$ E-6; Fig. 4).

\section{Other Clinical Variables}

Analgesic drug intake was decreased in four patients (33\%) and totally withdrawn in three additional patients (25\%) during the postoperative follow-up. All nine MCS responders $(100 \%$ of the MCS responders group, $75 \%$ of the entire population) preferred MCS to multiple rTMS sessions. One patient among the MCS nonresponders group (33\% of the MCS nonresponders group, $8.3 \%$ of the entire population) preferred MCS because of positive effects on motor performance. The other two MCS nonresponder patients (67\% of the MCS nonresponders group, $17 \%$ of the entire population) voiced no preference. A total of three patients (MCS responders cases 3 and 6 and MCS nonresponder case 5, 25\% of the entire population) reported positive motor effects with improved dexterity and fine movements of the hand contralateral to stimulation.

\section{MCS Adverse Side Effects}

Postoperative adverse side effects were observed in four patients (33\%): two $(17 \%)$ presented with hardware infection requiring complete removal of the neuromodulation devices, and one patient (8\%) presented with an aseptic 


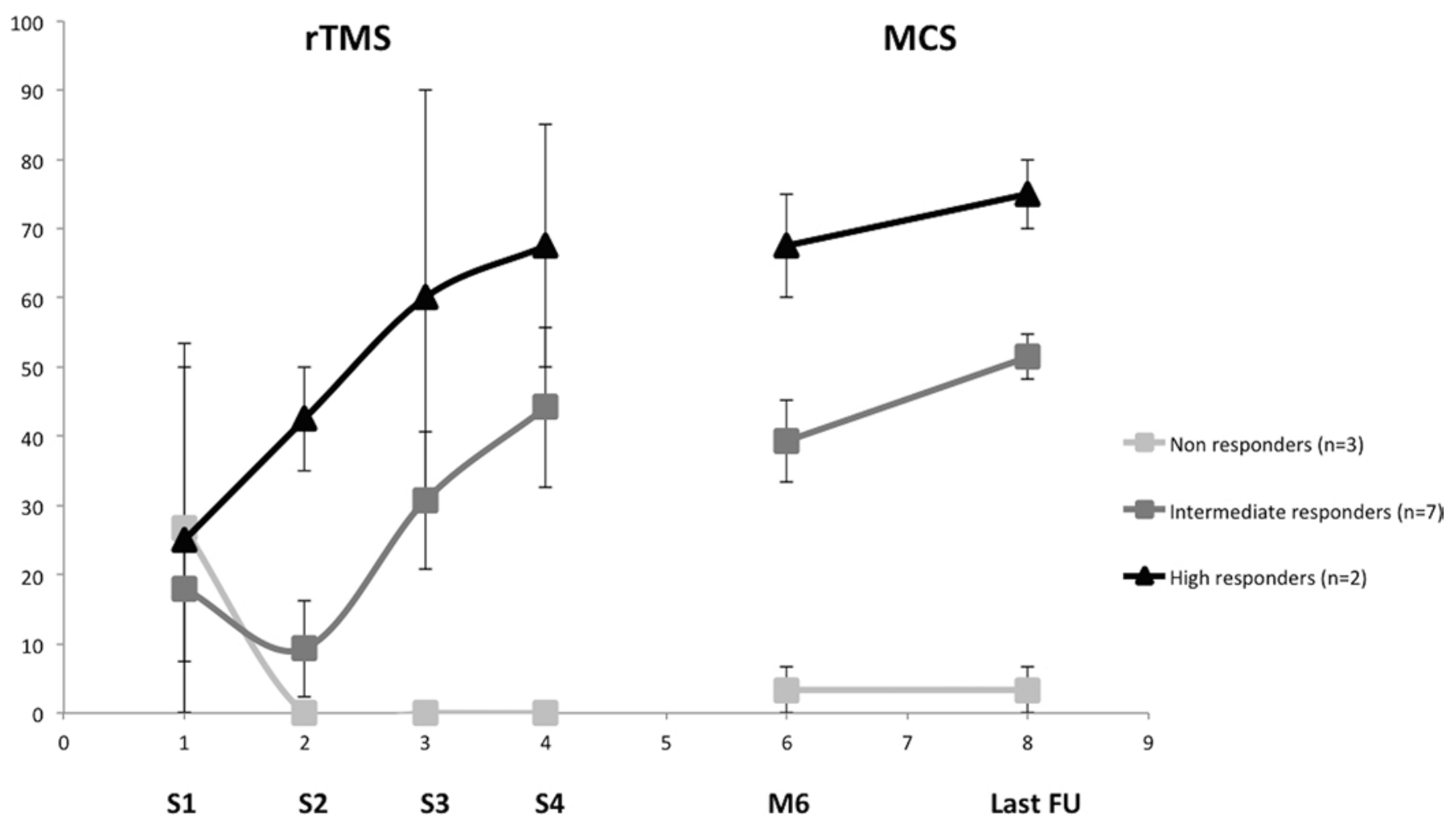

FIG. 3. Percentage of pain relief after rTMS and MCS in patients categorized according to their final \%R MCS (results are presented as mean \pm standard deviation). $\mathrm{FU}=$ follow-up; $\mathrm{M}=$ month; $\mathrm{S}=$ session.

wound dehiscence requiring removal of the neurostimulator alone. One patient $(8 \%)$ presented with a transient motor deficit caused by a limited intraparenchymal hemorrhage.

\section{Prediction of MCS Efficacy}

Linear Regression Models

The simple linear regression models showed no significant association between \% RTMS at the first session and \% R MCS at the last follow-up $\left(\mathrm{R}^{2}=0.004, \mathrm{p}=0.84\right)$, whereas it showed a significant association between \% R rTMS at the fourth session and \% MCS at the last followup $\left(\mathrm{R}^{2}=0.72, \mathrm{p}=0.0005\right)$. Likewise, the simple linear regression models showed no significant association between pain relief duration at the first rTMS session and \%R MCS at the last follow-up $\left(R^{2}=0.035, p=0.56\right)$, whereas it showed a significant association between pain relief dura-

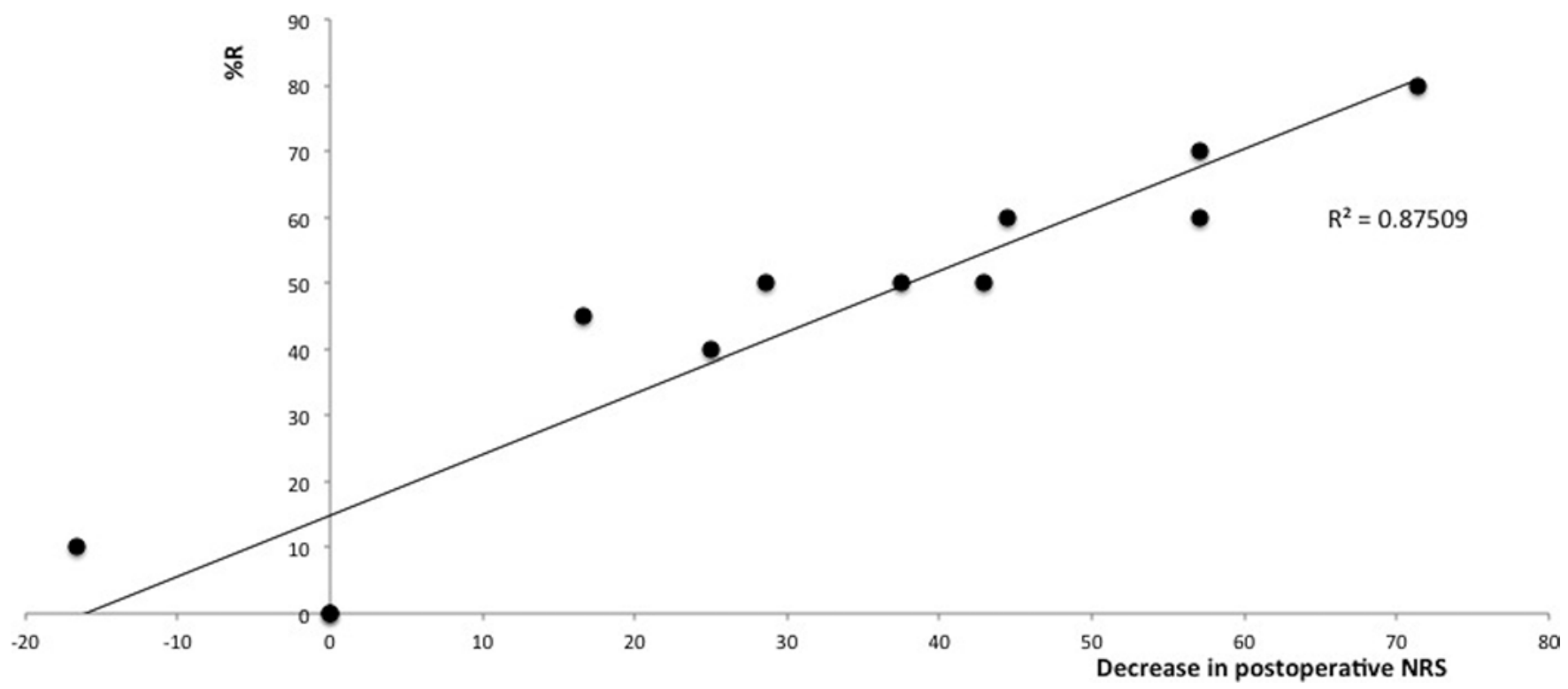

FIG. 4. Correlation observed between percentage of pain relief and percentage decrease of postoperative NRS $(R=0.94, p=$ 7.9E-6). 


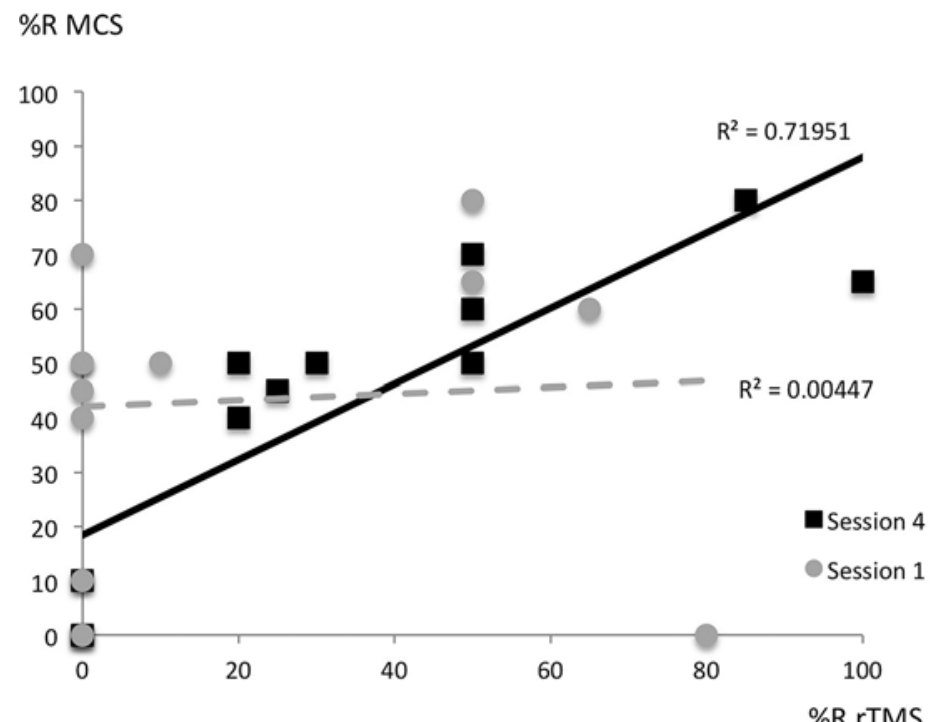

FIG. 5. Simple linear regressions models. Left: Association between \%R rTMS and \%R MCS. Right: Association between rTMS pain relief duration and \%R MCS. Gray circles correspond to the first rTMS session (nonsignificant results) and black squares to the fourth rTMS session (significant results).

tion at the fourth session and \% RCS at the last followup $\left(\mathrm{R}^{2}=0.47, \mathrm{p}=0.01\right.$; Fig. 5).

The multiple linear regression between $\% \mathrm{R}$ rTMS and pain relief duration at the fourth rTMS session (independent variables) and \% R MCS at the last follow-up (dependent variable) showed a significant association $\left(\mathrm{R}^{2}=0.83\right.$, $\mathrm{p}=0.0003$; Table 4).

\section{ROC Curve and Predictive Values}

Results were unchanged if the MCS success threshold was set to $30 \% \% \mathrm{R}$ MCS or $40 \% \% \mathrm{R}$ MCS. The ROC curve (Fig. 6) showed an area under the curve of 1 (SE: 0, CI: $1-1, p=0)$. At the fourth rTMS session, a \%R rTMS threshold set to $10 \%$ gave a sensitivity and specificity of $100 \%$. Youden's index was 1 . With these thresholds, all the rTMS responders demonstrated a response to MCS, whereas none of the rTMS nonresponders did so. With these thresholds, the PPV of rTMS at the fourth session was $100 \%$ (i.e., all the rTMS responders demonstrated a response to MCS) and the NPV was $100 \%$ (i.e., none of the rTMS nonresponders demonstrated a response to MCS; $\mathrm{p}$ $=0.0045)$. The results are presented in Fig. 7 .

\section{Other Clinical Variables}

A significant association was found between \% R rTMS at the fourth session and a decrease in analgesic drug intake at the last follow-up $(\mathrm{R}=0.77, \mathrm{p}=0.003)$.

\section{Discussion}

Motor cortex stimulation has been (empirically) discovered as a possible treatment for refractory neuropathic pain. ${ }^{35,36}$ However, despite a large number of case series $7,9,12,16,26,30,36$ and given the limited results from blind controlled studies, proof of efficacy has remained elusive. To date, more than 400 surgically treated patients have been reported on in the literature. Three main meta-analyses have described the results for 155 to 327 patients with an overall rate of $57 \%-65 \%$ of MCS responders ${ }^{13,24,28}$ (so a $35 \%-43 \%$ rate of failure). These findings highlight the lack of predictors to guide clinicians regarding patient eligibility for MCS, although several studies on this topic have been conducted. Integrity of the motor ${ }^{15}$ and sensory ${ }^{12}$ functions has been suggested as a positive predictor of MCS analgesic efficacy, but these findings were not replicated by Nuti et al. in a large series of 31 patients. ${ }^{30}$ The differential response to drugs such as ketamine, opioids, and barbiturates has also been investigated ${ }^{15}$ but subsequently challenged..$^{34}$ Finally, brain opioid receptor density, as assessed with ${ }^{11} \mathrm{C}$-diprenorphine positron emission tomography, could predict MCS efficacy, ${ }^{25}$ but it is hard to use both at the individual level and in clinical practice. To the best of our knowledge, a handful of studies (5) have investigated the correlation between rTMS- and MCS-induced pain relief after either single or multiple ${ }^{3,4,14,23,39}$ rTMS sessions. The

TABLE 4. Results of the multiple linear regression model

\begin{tabular}{lcccccc}
\hline \multicolumn{1}{c}{ Explicative Variable } & $\beta^{*}$ & $95 \% \mathrm{Cl}$ & Standardized $\beta$ & $95 \% \mathrm{Cl}$ & $\mathrm{R}^{2}$ & $\mathrm{p} \mathrm{Value \dagger}$ \\
\hline \%R at 4th rTMS session & 0.56 & $0.27-0.84$ & 0.68 & $0.33-1.03$ & 0.72 & 0.002 \\
\hline Pain relief duration at 4th rTMS session & 1.02 & $0.07-1.97$ & 0.37 & $0.03-0.72$ & 0.47 & 0.038 \\
\hline Model & & & & & 0.83 & 0.0003 \\
\hline
\end{tabular}

* Partial regression coefficient.

$\dagger$ Fisher test for each variable and for the overall model. 


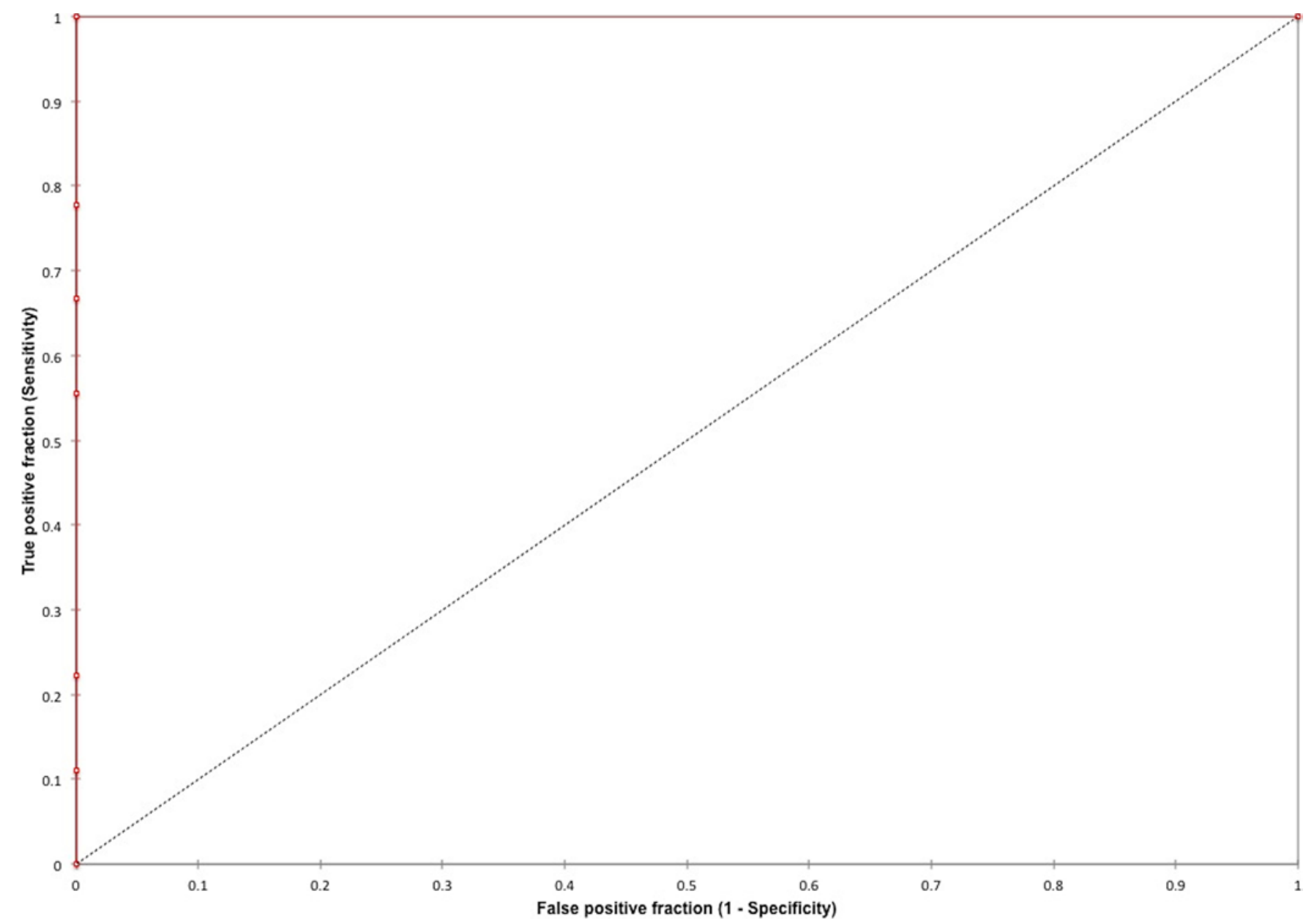

FIG. 6. Receiver operating characteristic (ROC) curve showing an area under the curve of 1 and an optimal \%R rTMS threshold of $10 \%$ at the fourth rTMS session. Figure is available in color online only.

first study ${ }^{14}$ considered only a single "active" rTMS session in 11 patients and showed a significant correlation between rTMS- and MCS-induced pain relief (visual analog scale [VAS] score reduction of $38.6 \%$ and $51.6 \%$, respectively). Three other single-rTMS-session studies ${ }^{3,4,23}$ used a crossover scheme with a sham rTMS session and extensively assessed the predictive value of rTMS. The PPV was excellent $(90 \%-100 \%)$, but the NPV was moderate $(65 \%-67 \%)$. In the report of André-Obadia et al., ${ }^{3}$ the NPV changed with the duration of MCS, with an increase in pain relief of 30\%-67\% between the onset of MCS and the 24-month follow-up. Only the study by Zhang et al..$^{39}$ investigated the contribution of multiple (i.e., 5-7) rTMS sessions, reporting a significant correlation with MCS success, but the protocol and clinical variables used to assess the predictive value were not detailed.

Interestingly, recently reported experiences with rTMS, ${ }^{17,33}$ including ours, ${ }^{33}$ have recommended multiple rTMS sessions to reach a plateau of analgesic effect. ${ }^{17,33}$ These findings led us to hypothesize that the correlation between rTMS and MCS might be advantageously assessed after several sessions of rTMS instead of a single one. In the present study, we set out to investigate the efficacy of rTMS during the first four sessions to be sure of reaching the plateau of pain relief.
Magnitude of pain relief was assessed by the declared percentage of pain relief (\%R TMS) as the main criterion. This variable has been proposed as a valuable tool to assess the efficacy of a therapy for chronic pain ${ }^{33}$ and has the advantage over other variables of having been commonly used for many years to evaluate pain relief in patients undergoing MCS. ${ }^{30}$ The present study did not include a placebo group for rTMS and MCS to assess this variable, which could be viewed as a limit. However, the efficacy of rTMS to treat neuropathic pain has been demonstrated in many studies, providing a high level of proof. ${ }^{19}$ In the same vein, MCS efficacy has been partially proven ${ }^{21,29,37}$ but suffers from the constant occurrence of unpredictable failures. Our main goal was to investigate the added value of multiple sessions of rTMS in more accurately predicting MCS outcomes in real-life clinical settings. Therefore, in our opinion, a placebo group was not an absolute requirement to assess a predictive value. We emphasize that our study does not constitute proof of efficacy for MCS (or for rTMS), which would need to be assessed through a large randomized controlled trial, but it does provide a clearer view of how to better select MCS candidates through preoperative screening with at least four rTMS sessions.

Consistent with our hypothesis of a cumulative effect at the initiation of rTMS, our results did not show any statisti- 

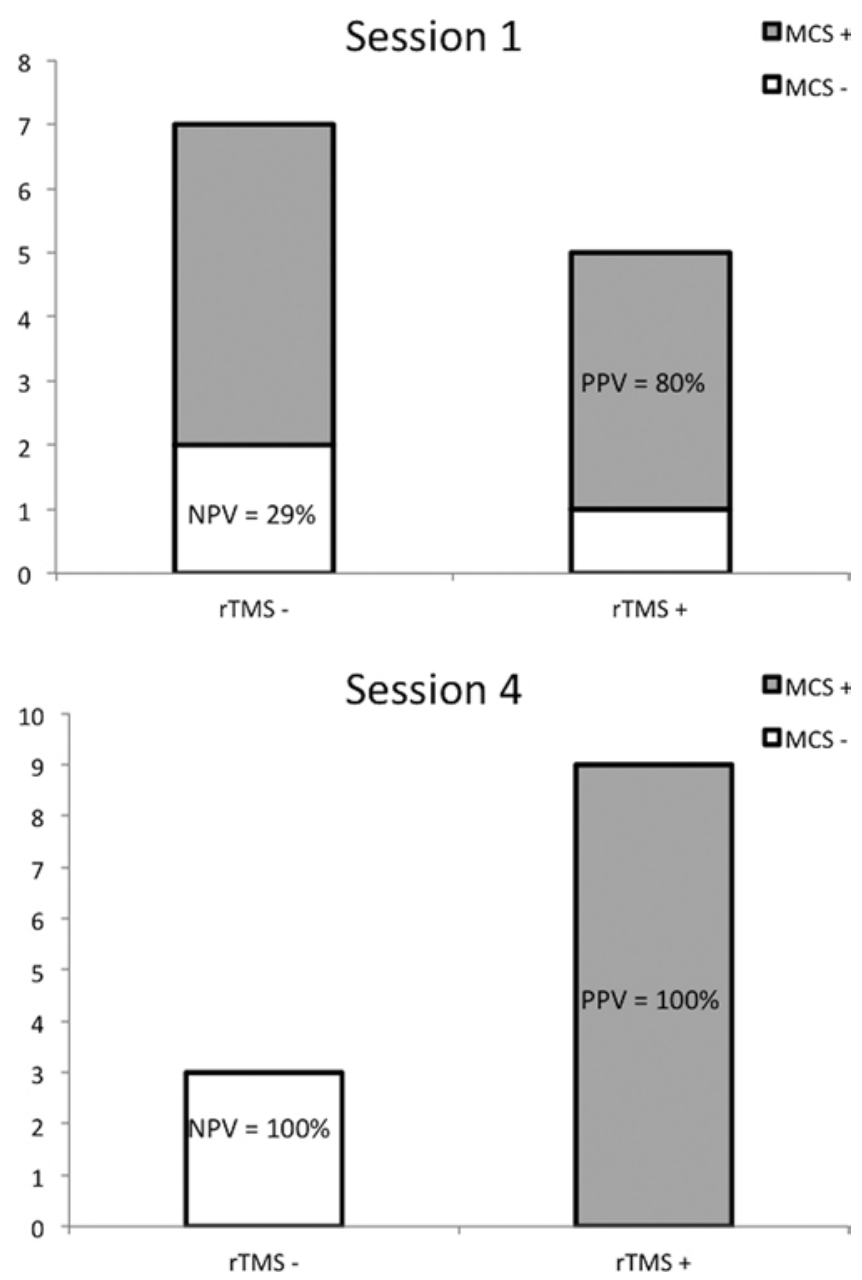

FIG. 7. Positive (PPV) and negative (NPV) predictive values of rTMS at the first (upper) and fourth (lower) rTMS sessions for MCS efficacy. Results are given considering a positive response threshold of $10 \% \mathrm{R}$ for rTMS (rTMS+) and 40\%R for MCS (MCS+; see text for details). Patients presenting with \%R lower than these scores are respectively represented as rTMS- and MCS-. The \%R rTMS at the fourth rTMS session provided statistically significant results $(p=0.0045)$ with high PPV and NPV. Instead of considering results at the fourth rTMS session, if we considered results after the first rTMS session, results were nonsignificant $(p=1)$ with a fairly high PPV of $80 \%$ but a low NPV of $29 \%$.

cal correlation between $\% \mathrm{R}$ rTMS at the first session and $\% \mathrm{R}$ MCS at the last follow-up, whereas the correlation was highly significant at the fourth rTMS session. This correlation was slightly more limited between the duration of pain relief at the fourth rTMS session and \% R MCS at the last follow-up, but these two variables used together allowed a high-quality multiple linear regression model with a coefficient of determination as high as $83 \%$. These results demonstrate a good prediction model for this population of patients and, for the first time, a strong incentive to investigate a larger population. In the same vein, contingency tables after the first rTMS session provided a PPV and an NPV of $80 \%$ and $29 \%$, respectively, which were good but a little lower than previous predictions. ${ }^{3,23}$ Possibly reflecting a fortunate coincidence if we consider the limited sample size of the population, the PPV and NPV nevertheless increased up to the fourth rTMS session, both reaching $100 \%$, thus highlighting the added value (through a cumulative analgesic effect) of multiple rTMS sessions to reach a valuable prediction of MCS efficacy. Finally, the contribution of multiple rTMS sessions as a strong predictor of MCS efficacy was further supported by the correlation between \%R rTMS at the fourth session and the decrease in drug intake after MCS (probably an even more representative indicator in terms of pain relief). Although the results were good to excellent, the conclusions that can be drawn from the statistical analysis must be interpreted in light of the limited sample size.

None of the patients judged rTMS to be a more effective treatment than MCS for their chronic pain. Among the nine rTMS responders subsequently operated on, all preferred MCS because they found the effect against pain more marked and stable, thus arguing in favor of surgery in such patients. One limit to surgery could be the iatrogenic risk. In the present study, up to $33 \%$ of patients suffered from adverse side effects. However, none of these effects was definitive, and we probably experienced an unfortunate coincidence, owing to the limited sample size, that does not match our previous experience with $\mathrm{MCS} .^{30}$ In a previous series of $31 \mathrm{MCS}$ patients, we reported a total complication rate of $26 \%$ including a large majority of rapidly resolving partial seizures. Moreover, in their literature review, Fontaine et al. reported only $5.7 \%$ of infections and $5.1 \%$ of hardware-related problems. ${ }^{13}$ Hence, patients should be informed about the risks of surgery, but the complication rate reported here seems not to reflect the real morbidity of MCS.

\section{Conclusions}

In summary, we propose that the use of multiple rTMS sessions (as opposed to a single one) can better predict MCS efficacy in patients suffering from severe refractory neuropathic pain. Currently, it seems the most valuable selection criterion available for offering patients MCS, with good prediction of both success and failure. Unpredictable failure remains the main restriction to proposing MCS; therefore, consistently predicting postoperative outcomes through noninvasive rTMS screening would greatly help in the surgery decision-making process. Further investigations conducted in larger cohorts and controlled trials are now needed to support our results and to continue moving toward a more efficient method for selecting MCS candidates.

\section{Acknowledgments}

We thank Dr. Edouard Ollier, MD, PhD (SAINBIOSE INSERM U1059, DVH Team, Université Jean Monnet, Saint-Etienne, France), for help with the statistical analysis.

\section{References}

1. André-Obadia N, Magnin M, Garcia-Larrea L: On the importance of placebo timing in rTMS studies for pain relief. Pain 152:1233-1237, 2011

2. André-Obadia N, Mertens P, Gueguen A, Peyron R, GarciaLarrea L: Pain relief by rTMS: differential effect of current 
flow but no specific action on pain subtypes. Neurology 71:833-840, 2008

3. André-Obadia N, Mertens P, Lelekov-Boissard T, Afif A, Magnin M, Garcia-Larrea L: Is life better after motor cortex stimulation for pain control? Results at long-term and their prediction by preoperative rTMS. Pain Physician 17:53-62, 2014

4. André-Obadia N, Peyron R, Mertens P, Mauguière F, Laurent B, Garcia-Larrea L: Transcranial magnetic stimulation for pain control. Double-blind study of different frequencies against placebo, and correlation with motor cortex stimulation efficacy. Clin Neurophysiol 117:1536-1544, 2006

5. Ayache SS, Ahdab R, Chalah MA, Farhat WH, Mylius V, Goujon C, et al: Analgesic effects of navigated motor cortex rTMS in patients with chronic neuropathic pain. Eur J Pain 20:1413-1422, 2016

6. Bouhassira D, Lantéri-Minet M, Attal N, Laurent B, Touboul C: Prevalence of chronic pain with neuropathic characteristics in the general population. Pain 136:380-387, 2008

7. Buchanan RJ, Darrow D, Monsivais D, Nadasdy Z, Gjini K: Motor cortex stimulation for neuropathic pain syndromes: a case series experience. Neuroreport 25:715-717, 2014

8. Canavero S, Bonicalzi V, Dotta M, Vighetti S, Asteggiano G, Cocito D: Transcranial magnetic cortical stimulation relieves central pain. Stereotact Funct Neurosurg 78:192-196, 2002

9. Carroll D, Joint C, Maartens N, Shlugman D, Stein J, Aziz TZ: Motor cortex stimulation for chronic neuropathic pain: a preliminary study of 10 cases. Pain 84:431-437, 2000

10. Demasles S, Peyron R, Garcia Larrea L, Laurent B: Les douleurs centrales post-AVC. Rev Neurol (Paris) 164:825-831, 2008

11. Dieleman JP, Kerklaan J, Huygen FJPM, Bouma PAD, Sturkenboom MCJM: Incidence rates and treatment of neuropathic pain conditions in the general population. Pain 137:681-688, 2008

12. Drouot X, Nguyen JP, Peschanski M, Lefaucheur JP: The antalgic efficacy of chronic motor cortex stimulation is related to sensory changes in the painful zone. Brain 125:1660 1664,2002

13. Fontaine D, Hamani C, Lozano A: Efficacy and safety of motor cortex stimulation for chronic neuropathic pain: critical review of the literature. J Neurosurg 110:251-256, 2009

14. Hosomi K, Saitoh Y, Kishima H, Oshino S, Hirata M, Tani N, et al: Electrical stimulation of primary motor cortex within the central sulcus for intractable neuropathic pain. Clin Neurophysiol 119:993-1001, 2008

15. Katayama Y, Fukaya C, Yamamoto T: Poststroke pain control by chronic motor cortex stimulation: neurological characteristics predicting a favorable response. J Neurosurg 89:585591, 1998

16. Katayama Y, Tsubokawa T, Yamamoto T: Chronic motor cortex stimulation for central deafferentation pain: experience with bulbar pain secondary to Wallenberg syndrome. Stereotact Funct Neurosurg 62:295-299, 1994

17. Kobayashi M, Fujimaki T, Mihara B, Ohira T: Repetitive transcranial magnetic stimulation once a week induces sustainable long-term relief of central poststroke pain. Neuromodulation 18:249-254, 2015

18. Lee JJ, Lee MK, Kim JE, Kim HZ, Park SH, Tae JH, et al: Pain relief scale is more highly correlated with numerical rating scale than with visual analogue scale in chronic pain patients. Pain Physician 18:E195-E200, 2015

19. Lefaucheur JP, André-Obadia N, Antal A, Ayache SS, Baeken C, Benninger DH, et al: Evidence-based guidelines on the therapeutic use of repetitive transcranial magnetic stimulation (rTMS). Clin Neurophysiol 125:2150-2206, 2014

20. Lefaucheur JP, André-Obadia N, Poulet E, Devanne H, Haffen E, Londero A, et al: Recommandations françaises sur l'utilisation de la stimulation magnétique transcrânienne répétitive (rTMS) : règles de sécurité et indications thérapeutiques. Neurophysiol Clin 41:221-295, 2011

21. Lefaucheur JP, Drouot X, Cunin P, Bruckert R, Lepetit H, Créange A, et al: Motor cortex stimulation for the treatment of refractory peripheral neuropathic pain. Brain 132:14631471,2009

22. Lefaucheur JP, Drouot X, Keravel Y, Nguyen JP: Pain relief induced by repetitive transcranial magnetic stimulation of precentral cortex. Neuroreport 12:2963-2965, 2001

23. Lefaucheur JP, Ménard-Lefaucheur I, Goujon C, Keravel Y, Nguyen JP: Predictive value of rTMS in the identification of responders to epidural motor cortex stimulation therapy for pain. J Pain 12:1102-1111, 2011

24. Lima MC, Fregni F: Motor cortex stimulation for chronic pain: systematic review and meta-analysis of the literature. Neurology 70:2329-2337, 2008

25. Maarrawi J, Peyron R, Mertens P, Costes N, Magnin M, Sindou M, et al: Brain opioid receptor density predicts motor cortex stimulation efficacy for chronic pain. Pain 154:25632568, 2013

26. Mertens P, Nuti C, Sindou M, Guenot M, Peyron R, GarciaLarrea L, et al: Precentral cortex stimulation for the treatment of central neuropathic pain: results of a prospective study in a 20-patient series. Stereotact Funct Neurosurg 73:122-125, 1999

27. Mhalla A, Baudic S, Ciampi de Andrade D, Gautron M, Perrot S, Teixeira MJ, et al: Long-term maintenance of the analgesic effects of transcranial magnetic stimulation in fibromyalgia. Pain 152:1478-1485, 2011

28. Nguyen JP, Lefaucheur JP, Raoul S, Roualdes V, Péréon Y, Keravel Y: Motor cortex stimulation for the treatment of neuropathic pain, in Krames ES, Pecham PH, Rezai AR (eds): Neuromodulation. London: Academic Press, 2009, pp $515-526$

29. Nguyen JP, Velasco F, Brugières P, Velasco M, Keravel Y, Boleaga B, et al: Treatment of chronic neuropathic pain by motor cortex stimulation: results of a bicentric controlled crossover trial. Brain Stimul 1:89-96, 2008

30. Nuti C, Peyron R, Garcia-Larrea L, Brunon J, Laurent B, Sindou M, et al: Motor cortex stimulation for refractory neuropathic pain: four year outcome and predictors of efficacy. Pain 118:43-52, 2005

31. Passard A, Attal N, Benadhira R, Brasseur L, Saba G, Sichere $\mathrm{P}$, et al: Effects of unilateral repetitive transcranial magnetic stimulation of the motor cortex on chronic widespread pain in fibromyalgia. Brain 130:2661-2670, 2007

32. Penfield W, Boldrey E: Somatic motor and sensory representation in the cerebral cortex of man as studied by electrical stimulation. Brain J Neurol 60:389-443, 1937

33. Pommier B, Créac'h C, Beauvieux V, Nuti C, Vassal F, Peyron R: Robot-guided neuronavigated rTMS as an alternative therapy for central (neuropathic) pain: Clinical experience and long-term follow-up. Eur J Pain 20:907-916, 2016

34. Saitoh Y, Kato A, Ninomiya H, Baba T, Shibata M, Mashimo T, et al: Primary motor cortex stimulation within the central sulcus for treating deafferentation pain. Acta Neurochir Suppl 87:149-152, 2003

35. Tsubokawa T, Katayama Y, Yamamoto T, Hirayama T, Koyama S: Chronic motor cortex stimulation for the treatment of central pain. Acta Neurochir Suppl (Wien) 52:137-139, 1991

36. Tsubokawa T, Katayama Y, Yamamoto T, Hirayama T, Koyama S: Treatment of thalamic pain by chronic motor cortex stimulation. Pacing Clin Electrophysiol 14:131-134, 1991

37. Velasco F, Argüelles C, Carrillo-Ruiz JD, Castro G, Velasco AL, Jiménez F, et al: Efficacy of motor cortex stimulation in the treatment of neuropathic pain: a randomized double-blind trial. J Neurosurg 108:698-706, 2008 
38. Yamamoto T, Katayama Y, Hirayama T, Tsubokawa T: Pharmacological classification of central post-stroke pain: comparison with the results of chronic motor cortex stimulation therapy. Pain 72:5-12, 1997

39. Zhang X, Hu Y, Tao W, Zhu H, Xiao D, Li Y: The effect of motor cortex stimulation on central poststroke pain in a series of 16 patients with a mean follow-up of 28 months: the effect and outcome predictors of MCS. Neuromodul Technol Neural Interface 20:492-496, 2017

\section{Disclosures}

The authors report no conflict of interest concerning the materials or methods used in this study or the findings specified in this paper.

\section{Author Contributions}

Conception and design: Pommier, Vassal, Peyron. Acquisition of data: Pommier, Quesada, Nuti, Vassal, Peyron. Analysis and interpretation of data: Pommier, Quesada, Vassal, Peyron. Drafting the article: Pommier. Critically revising the article: Quesada, Fauchon, Vassal, Peyron. Approved the final version of the manuscript on behalf of all authors: Pommier. Administrative/techni$\mathrm{cal} /$ material support: Vassal, Peyron.

\section{Supplemental Information}

Online-Only Content

Supplemental material is available with the online version of the article.

Supplementary Fig. 1. https://thejns.org/doi/suppl/10.3171/ 2017.12.JNS171333.

\section{Correspondence}

Benjamin Pommier: Centre Hospitalier Régional Universitaire de Saint-Etienne, Hôpital Nord, Saint-Priest-en-Jarez, France. pommier.benjamin05@gmail.com. 